\section{The American Association for Thoracic Surgery Volunteer Leadership and Senior Staff Disclosure Statement}

To best serve the interests of the specialty and its practitioners, the American Association for Thoracic Surgery requires the volunteer leadership, including its editors, asso- ciate editors, and section editors, to publicly disclose the existence and nature of any financial or other relationships that might influence, or appear to influence, their actions. In keeping with this policy, the following disclosures have been made by the editors and editorial board members responsible for making decisions about manuscripts submitted to and published in the official publications of the Association:

\begin{tabular}{ll}
\hline $\begin{array}{l}\text { Lawrence Cohn } \\
\text { Cary Akins }\end{array}$ & $\begin{array}{l}\text { JTCVS Editor } \\
\text { JTCVS Associate Editor }\end{array}$ \\
$\begin{array}{l}\text { Emile Bacha } \\
\text { Eugene Blackstone }\end{array}$ & $\begin{array}{l}\text { JTCVS Associate Editor } \\
\text { JTCVS Associate Editor }\end{array}$ \\
Christopher Caldarone & JTCVS Associate Editor \\
Fred Crawford & Op Techs Editor \\
Harvey Pass & Seminars Editor
\end{tabular}

Todd Rosengart Joseph Dearani Jennifer Lawton William Williams Niv Ad Sary Aranki Erle Austin Carl Backer Vinay Badhwar Leora Balsam Munir Boodhwani Michael Borger

Scott Bradley Duke Cameron Andrea Carpenter Thierry-Pierre Carrel Robert Cerfolio

Fred Chen

Yolanda Colson Michael D'Ambra Hiroshi Date William DeCampli Chad Denlinger John Elefteriades

James Fann Victor Ferraris

Daniel Fitzgerald

Raja Flores

Charles Fraser
Seminars Editor

Pediatrics Annual Editor/JTCVS Editorial Board TSN Editor

Associate Editor, Thoracic Surgery News JTCVS Editorial Board JTCVS Editorial Board JTCVS Editorial Board JTCVS Editorial Board JTCVS Editorial Board JTCVS Editorial Board JTCVS Editorial Board JTCVS Editorial Board

JTCVS Editorial Board JTCVS Editorial Board JTCVS Editorial Board JTCVS Editorial Board JTCVS Editorial Board

JTCVS Editorial Board JTCVS Editorial Board JTCVS Editorial Board JTCVS Editorial Board JTCVS Editorial Board JTCVS Editorial Board JTCVS Editorial Board

JTCVS Editorial Board JTCVS Editorial Board

JTCVS Editorial Board JTCVS Editorial Board JTCVS Editorial Board
Nothing to Disclose

Metronic Inc, Licensed Patent for a Perfusion Cannula; Edwards LifeSciences, Data Safety Monitoring Board Member

Cormatrix, Speaker

Churchill-Livingstone Book Royalties, Author; Edwards LifeSciences, Partner Publication Office

CellAegis Inc, Ownership

Medronic, Stock; Johnson and Johnson, Stock

Champions Oncology, Advisory Board; General Electric, Advisory Board; Intergrated Diagnostics, Research Collaborator; National Cancer Instutute, Research Funding; Belluck and Fox, Philanthropy; Levi, Phillips, and Konigsberg, Philanthropy; Pinpoint Genomics, Advisory Board; Celera, Research Collaborator; Somalogic, Research Collaborator; GSK, Advisory Panel; Quest, Advisory Board; Transgenomics, Research Funding

Nothing to Disclose

Nothing to Disclose

Nothing to Disclose

Nothing to Disclose

Metronic, Consultant; Estech, Consultant; Atricure, Consultant

Nothing to Disclose

Nothing to Disclose

Nothing to Disclose

Nothing to Disclose

Nothing to Disclose

Nothing to Disclose

Edwards Lifesciences, Speakers; Medtronic, Speaker; St. Jude Medical, Speaker

Nothing to Disclose

Nothing to Disclose

Nothing to Disclose

Nothing to Disclose

Intuitive Surgical, Faculty; Pfizer, Research Grant; Neomend, Research Grant; Precision Therapeutics, Research Grant

Nothing to Disclose

Nothing to Disclose

PeriCor Therapeutics; Board of Directors; D'Ambra Technologies, Partner

Nothing to Disclose

Nothing to Disclose

Nothing to Disclose

Covidien, Consultant; Datascope, Consultant; CoolSpine, Medical Director; Medtronic, PI

Nothing to Disclose

Haemonetics, Advisory Board; Baxter HealthCare, CME Event; NovoNordisk, Consultant; Astra Zeneca, CME Event \& Advisory

Nothing to Disclose

Nothing to Disclose

Nothing to Disclose 
J. William Gaynor

Donald Glower

Alden Harken

Shen-shou Hu

John Ikonomidis

Kirk Kanter

Nevin Katz

Ben Kozower

Irving Kron

Paul Kurlansky

Jennifer Lawton

Harold Lazar

Michael Liptay

Audrey Marshall

Patrick McCarthy

P. Michael McFadden

Carlos Mestres

Marc Moon

Christine Mora-Mangano

Sudish Murthy

Bryan Meyers

Katie Nason

Thomas Ng

Takashi Nishimura

Soon Park

Frank Pigula

Thomas Rice

Hans-Joachim Schaefers

Hartzell Schaff

Frank Sellke

Oz Shapira

Richard Shemin

Edward Soltesz

Rakesh Suri

Lars Svensson

John Toole

Dirk Van Raemdonck

Federico Venuta

Jakob Vinten-Johansen

Ara Vaporciyan

Y. Joseph Woo

Stephen Yang

Richard Weisel

Mara Antonoff

Sanford Zeigler
JTCVS Editorial Board

JTCVS Editorial Board JTCVS Editorial Board JTCVS Editorial Board JTCVS Associate Editor JTCVS Editorial Board JTCVS Associate Editor JTCVS Editorial Board JTCVS Editorial Board JTCVS Editorial Board JTCVS Editorial Board JTCVS Editorial Board JTCVS Editorial Board JTCVS Editorial Board JTCVS Editorial Board

JTCVS Editorial Board JTCVS Editorial Board

JTCVS Editorial Board JTCVS Editorial Board JTCVS Editorial Board JTCVS Associate Editor

JTCVS Editorial Board JTCVS Editorial Board JTCVS Editorial Board JTCVS Editorial Board

JTCVS Editorial Board JTCVS Associate Editor JTCVS Editorial Board JTCVS Associate Editor

JTCVS Associate Editor

JTCVS Editorial Board JTCVS Editorial Board JTCVS Editorial Board JTCVS Editorial Board

JTCVS Editorial Board JTCVS Editorial Board JTCVS Editorial Board JTCVS Editorial Board JTCVS Editorial Board JTCVS Associate Editor JTCVS Editorial Board JTCVS Editorial Board JTCVS Editorial Board TSRA Associate Editor, Thoracic Surgery News TSRA Associate Editor, Thoracic Surgery News
Johnson and Johnson, Stock; Bristol Myers Squibb, Stock; Medtronic, Stock; Pfizer, Stock; General Electric, Bonds and Stock; Lilly; Stock; Merck, Stock; Dow, Stock; Dupont, Stock

Nothing to Disclose

Nothing to Disclose

Nothing to Disclose

Nothing to Disclose

Nothing to Disclose

Nothing to Disclose

Nothing to Disclose

Nothing to Disclose

Nothing to Disclose

Nothing to Disclose

Eli Lilly Inc, Research Grant

Covidien, Proctor and Course Director

Nothing to Disclose

Edwards Lifesciences LLC, Intellectual Property and Consulting; Cardious Inc, Consultant; Baxter, Advisory Board; Abbott, Consultant; Micardia, Consultant; Direct Flow; Travel

Nothing to Disclose

Novartis Pharma, Advisory Board; Edwards Lifescience, Consultant; Symetis SA, Advisory Board

Nothing to Disclose

Pericor Inc, Owner; ATGC, Owner

Nothing to Disclose

Ethicon Endo-Surgery, Speaker, Teacher, Consultant; Varian, Speaker, Consultant

Nothing to Disclose

Nothing to Disclose

Nothing to Disclose

Thoratec, Education, Research, and Consulting; Cleveland Clinic, Consultant

Nothing to Disclose

Nothing to Disclose

Nothing to Disclose

St. Jude Medical, PI on Valve Study; Edwards, Consultant, Paricipant in Prtner Trial; Kips Bay Medical, Participant in Emesh Trial; AtriCure, Consultant

Medicines Company, Advisory Board; CLS Behring, Adjudication Committee

Nothing to Disclose

Edwards Lifesciences, Consultant; AtriCure, Consultant

St. Jude Medical, Speaker; TransMedics, Steering Committee Member

Edward Lifesciences, Research Grant; Sorin, Research Grant, PI, IP; St. Jude Medical, Research Grant, Steering Committee

Cardiosolutions; Posthorax; ValveXchange

Nothing to Disclose

Nothing to Disclose

Nothing to Disclose

Nothing to Dsclose

Nothing to Disclose

Nothing to Disclose

Nothing to Disclose

Nothing to Disclose

Nothing to Disclose

Nothing to Disclose 